\title{
Area at End Ventricular Diastole
}

National Cancer Institute

\section{Source}

National Cancer Institute. Area at End Ventricular Diastole. NCI Thesaurus. Code C127537.

The 2-dimensional surface enclosed within a boundary and measured at end ventricular diastole. 